\title{
Para-orthogonal polynomials and chain sequences
}

\author{
C.F. Bracciali, A. Sri Ranga, \\ Depto de Matemática Aplicada, IBILCE, UNESP, \\ 15054-000, São José do Rio Preto, SP, Brasil \\ cleonice@ibilce.unesp.br, ranga@ibilce.unesp.br
}

\author{
A. Swaminathan, \\ Dept. of Mathematics, IIT Roorkee, \\ Roorkee, India \\ mathswami@gmail.com
}

Resumo: Three term recurrence formula has been an important tool in the studies of orthogonal polynomials on the real line. Positive chain sequences also play an important role in these studies. In recent years we have realized that the use of a different type of three term recurrence formula in combination with the use of positive chain sequences is also very important for studying the properties of orthogonal polynomials on the unit circle. In this text we give some new connection that exists between orthogonal polynomials on the unit circle and para-orthogonal polynomials given by three term recurrence formula.

Palavras-chave: Orthogonal polynomials on the unit circle, Three term recurrence formula, Positive chain sequences

\section{Introduction}

One of the important properties of orthogonal polynomials on the real line (see $[3,6,8]$ ) is that they satisfy a three term recurrence formula. This property has been thoroughly explored by many for studying the properties of these polynomials and positive chain sequences have been a important ally in this exploration.

Following the definition adopted by Chihara [3], we say that $\left\{d_{n}\right\}_{n=1}^{\infty}$ is a positive chain sequence if there exists a second sequence $\left\{g_{n}\right\}_{n=0}^{\infty}$ such that

$$
\begin{aligned}
& \text { i) } \quad 0 \leq g_{0}<1, \quad 0<g_{n}<1 \text { for } n \geq 1 \text {; } \\
& \text { ii) } \quad d_{n}=\left(1-g_{n-1}\right) g_{n} \text { for } n \geq 1 .
\end{aligned}
$$

This definition is slightly more restrictive than the definition used for general chain sequences by Wall [9], where any of the $g_{n}(n \geq 1)$ is also allowed to take the value zero.

The sequence $\left\{g_{n}\right\}_{n=0}^{\infty}$ is called a parameter sequence of the positive chain sequence $\left\{d_{n}\right\}_{n=1}^{\infty}$. In general the parameter sequence of a positive chain sequence is not unique. Every positive chain sequence has a minimal parameter sequence $\left\{m_{n}\right\}_{n=0}^{\infty}$ uniquely determined by the condition $m_{0}=0$. Every positive chain sequence $\left\{d_{n}\right\}_{n=1}^{\infty}$ also has a maximal parameter sequence $\left\{M_{n}\right\}_{n=0}^{\infty}$ which is characterized by the condition if $g_{0}>M_{0}$ then $\left\{g_{n}\right\}_{n=0}^{\infty}$ generated by (ii) of (1.1) does not satisfy (i) of (1.1). Wall's criteria for $\left\{M_{n}\right\}_{n=0}^{\infty}$ to be the maximal parameter sequence is

$$
\sum_{n=1}^{\infty} \frac{M_{1} M_{2} \cdots M_{n}}{\left(1-M_{1}\right)\left(1-M_{2}\right) \cdots\left(1-M_{n}\right)}=\infty .
$$

If the maximal parameter sequence of a chain sequence coincide with its minimal parameter sequence, then we say that the chain sequence is a single parameter chain sequence. 
In recent years we have realized that a different type of three term recurrence formula, combined with positive chain sequences, is also a very useful tool for studying the properties of orthogonal polynomials on the unit circle (OPUC). Applications that stem from these studies include quadrature formulas on the unit circle, which we will not be treating in the present text.

The three term recurrence formula in our studies is the following.

$$
R_{n+1}(z)=\left[\left(1+i c_{n+1}\right) z+\left(1-i c_{n+1}\right)\right] R_{n}(z)-4 d_{n+1} z R_{n-1}(z), \quad n \geq 1,
$$

with $R_{0}(z)=1$ and $R_{1}(z)=\left(1+i c_{1}\right) z+\left(1-i c_{1}\right)$, where

and

$$
\left\{c_{n}\right\}_{n=1}^{\infty} \text { is a sequence of real numbers }
$$

$$
\left\{d_{n}\right\}_{n=1}^{\infty} \text { is a positive chain sequence. }
$$

The following result was established in [5].

Theorem 1.1 [5] The polynomial $R_{n}$ has $n$ simple zeros on the open unit circle $|z|=1, z \neq 1$, and denoting these zeros by $e^{i \theta_{n, j}}, j=1,2, \ldots, n$, then there holds the interlacing property

$$
0<\theta_{n+1,1}<\theta_{n, 1}<\theta_{n+1,2}<\cdots<\theta_{n, n}<\theta_{n+1, n+1}<2 \pi,
$$

for $n \geq 1$. Moreover,

a) if $c_{k}>0$ for $1 \leq k \leq n$ and $\check{c}_{n}=\min _{1 \leq k \leq n} c_{k}$ then

$$
\theta_{n, n}<2 \pi-2 \arccos \left(\frac{1}{\sqrt{1+\check{c}_{n}^{2}}}\right) ;
$$

b) if $c_{k}<0$ for $1 \leq k \leq n$ and $\hat{c}_{n}=\max _{1 \leq k \leq n} c_{k}$ then

$$
2 \arccos \left(\frac{1}{\sqrt{1+\hat{c}_{n}^{2}}}\right)<\theta_{n, 1}
$$

The above fact that the zeros of $R_{n}$ are simple and that they interlace with the zeros of $R_{n+1}$ is very important for obtaining the results given in this text. We remark that this fact is also important from the point of view of obtaining positive quadrature formulas on the unit circle which are equivalent to the Gaussian quadrature formulas on the real line.

In terms of a certain sequence of OPUC, say $\left\{S_{n}\right\}$, the polynomials $R_{n}$ can be given by the combination $S_{n}(z)+\tau_{n} S_{n}^{*}(z)$, where $\tau_{n}$ is such that $\left|\tau_{n}\right|=1$ and $S_{n}^{*}(z)=z^{n} \overline{S_{n}(1 / \bar{z})}$ are the reciprocal polynomials (see equation (2.5) in the present text). Combinations of the type $S_{n}(z)+\tau_{n} S_{n}^{*}(z)$ are known in the literature of OPUC as para-orthogonal polynomials on the unit circle. Thus, the choice of the name para-orthogonal in the title of this text.

\section{$2 \quad$ Associated moment functional and measures}

In [4] and [2] it was established that associated with the above three term recurrence formula one can associate a moment functional $\mathcal{N}$ such that the polynomials $\left\{R_{n}\right\}$ satisfy the following orthogonality property.

$$
\mathcal{N}\left[z^{-n+k} R_{n}(z)\right]=0, \quad k=0,1, \ldots, n-1 .
$$

Moreover, with an appropriate choice of an additional element $d_{1}$ to the positive chain sequence $\left\{d_{n+1}\right\}_{n=1}^{\infty}$, one can also associate a positive measure $\tilde{\mu}$ on the unit circle such that

$$
\mathcal{N}[\ell(z)]=\int_{\mathcal{C}} \ell(\zeta)(1-\zeta) d \tilde{\mu}(\zeta)
$$

The objective of this text is to present another positive measure that can be associated with the above three term recurrence formula and, in particular, to give the following result. 
Theorem 2.1 Given the three term recurrence formula (1.2) there exists a nontrivial probability measure $\hat{\mu}$ on the unit circle such that for any Laurent polynomial $\ell$,

$$
\int_{\mathcal{C}} \ell(\zeta) d \hat{\mu}(\zeta)=\frac{1+c_{1}^{2}}{4 d_{1}} \mathcal{N}\left[\ell(z)\left(1-z^{-1}\right)\right]
$$

Here, $\mathcal{N}$ is the moment functional stated above. The sequence of monic OPUC $\left\{\hat{S}_{n}\right\}$ with respect to the measure $\hat{\mu}$ are

$$
\hat{S}_{n}(z)=\frac{R_{n+1}(z)-2\left(1-\mathfrak{m}_{n}\right) R_{n}(z)}{(z-1) \prod_{k=1}^{n+1}\left(1+i c_{k}\right)}, \quad n \geq 0 .
$$

Here, $\left\{\mathfrak{m}_{n}\right\}_{n=0}^{\infty}$, where $\mathfrak{m}_{n}=1-\frac{R_{n+1}(1)}{2 R_{n}(1)}, n \geq 0$, is the minimal parameter sequence of $\left\{d_{n+1}\right\}_{n=1}^{\infty}$. If the measure $\hat{\mu}$ is such that the principal value integral $I=f_{\mathcal{C}} \zeta(\zeta-1)^{-1} d \hat{\mu}(\zeta)$ exists then

$$
\frac{1+c_{1}^{2}}{4 d_{1}} \mathcal{N}[\ell(z)]=f_{\mathcal{C}} \ell(\zeta) \frac{\zeta}{\zeta-1} d \hat{\mu}(\zeta)+i t \ell(1)
$$

where $t=-\mathcal{I} m\left[\left(1+i c_{1}\right) I\right]$. In particular, if the positive chain sequence $\left\{d_{n+1}\right\}_{n=1}^{\infty}$ is not a single parameter chain sequence then the measure $\hat{\mu}$ is such that $\mathcal{I} m\left[\left(1+i c_{1}\right) I\right]=0$.

Proof. The proof of this Theorem is rather extensive. It is based on the correspondence and convergence properties of the rational functions

$$
\frac{\left(1+i c_{1}\right)}{2 z R_{n}(z)}\left[R_{n}(z)+\frac{\left(1-i c_{1}\right)}{2 d_{1}}(z-1) Q_{n}(z)\right], \quad n \geq 1,
$$

where the polynomials $\left\{Q_{n}\right\}$ satisfy the same recurrence relation (1.2), but with the initial conditions $Q_{0}(z)=0$ and $Q_{1}(z)=2 d_{1}$. Here, $d_{1} \neq 0$. It is crucially important to our analysis that the zeros of the polynomials $R_{n}$ are as established in Theorem 1.1. The idea behind the proof is similar to the one given in [2], where the analysis were based on the correspondence and convergence properties of the rational functions $\frac{R_{n}(z)-Q_{n}(z)}{(z-1) R_{n}(z)}, n \geq 1$.

Example given in Section 3 to illustrate the above theorem is also of great importance because of its explicit nature.

Since the polynomials $R_{n}$ are such that $R_{n}^{*}(z)=z^{n} \overline{R_{n}(1 / \bar{z})}=R_{n}(z), n \geq 0$, we also have from Theorem 2.1 that

$$
\hat{S}_{n}^{*}(z)=\frac{R_{n+1}(z)-2\left(1-\mathfrak{m}_{n}\right) z R_{n}(z)}{(1-z) \prod_{k=1}^{n+1}\left(1-i c_{k}\right)}, \quad n \geq 0 .
$$

By elimination of $R_{n+1}$ using (2.3) and (2.4),

$$
\frac{1}{\prod_{k=1}^{n}\left(1+i c_{k}\right)} R_{n}(z)=\frac{1+i c_{n+1}}{2\left(1-\mathfrak{m}_{n}\right)}\left[\hat{S}_{n}(z)+\tau_{n+1} \hat{S}_{n}^{*}(z)\right], \quad n \geq 0
$$

where $\tau_{n}=\prod_{n=1}^{n} \frac{1-i c_{n}}{1+i c_{n}}, n \geq 1$. Similarly, by elimination of $R_{n}$ using (2.3) and (2.4),

$$
\frac{1}{\prod_{k=1}^{n+1}\left(1+i c_{k}\right)} R_{n+1}(z)=z \hat{S}_{n}(z)+\tau_{n+1} \hat{S}_{n}^{*}(z), \quad n \geq 0 .
$$

Observe that the polynomials appearing on both sides of (2.5) and (2.6) are monic polynomials. Hence, we can also write (2.5) in the form

$$
\frac{1}{\prod_{k=1}^{n}\left(1+i c_{k}\right)} R_{n}(z)=\frac{1}{1-\tau_{n+1} \hat{\alpha}_{n-1}}\left[\hat{S}_{n}(z)+\tau_{n+1} \hat{S}_{n}^{*}(z)\right], \quad n \geq 0 .
$$


Moreover, using in the above expression the well known two term recurrence formula for the OPUC $\hat{S}_{n}$

$$
\frac{1}{\prod_{k=1}^{n}\left(1+i c_{k}\right)} R_{n}(z)=\left[z \hat{S}_{n-1}(z)+\frac{\tau_{n+1}-\overline{\hat{\alpha}_{n-1}}}{1-\tau_{n+1} \hat{\alpha}_{n-1}} \hat{S}_{n-1}^{*}(z)\right], \quad n \geq 1 .
$$

Here, $\alpha_{n-1}=-\overline{\hat{S}_{n}(0)}, n \geq 1$ are known as the Verblunsky coefficients associated with the measure $\hat{\mu}$. Comparing the above formula for $R_{n}$ with $(2.6)$ we have $\tau_{n}=\frac{\tau_{n+1}-\bar{\alpha}_{n-1}}{1-\tau_{n+1} \hat{\alpha}_{n-1}}, n \geq 1$, or equivalently,

$$
\tau_{n+1}=\frac{\tau_{n}+\overline{\hat{\alpha}_{n-1}}}{1+\tau_{n} \hat{\alpha}_{n-1}}, \quad n \geq 1 .
$$

The above recurrence formula for $\tau_{n}$ is useful if we can find an expression for $\tau_{1}$ in terms of the measure $\hat{\mu}$.

Assuming $I=f_{\mathcal{C}}(\zeta-1)^{-1} \zeta d \hat{\mu}(\zeta)$ exists, from $\mathcal{N}\left[z^{-1} R_{1}(z)\right]=0$ and $\tau_{1}=\left(1-i c_{1}\right) /\left(1+i c_{1}\right)$, the required expression for $\tau_{1}$ is

$$
\tau_{1}=\frac{I+i t}{\bar{I}-i t} .
$$

From $\tau_{n}=\prod_{k=1}^{n}\left(1-i c_{k}\right) /\left(1+i c_{k}\right), n \geq 1$, and the recurrence relation for $\left\{\tau_{n}\right\}$,

$$
c_{1}=i \frac{\tau_{1}-1}{\tau_{1}+1}, \quad c_{n+1}=\frac{\mathcal{I} m\left(\tau_{n+1} \hat{\alpha}_{n-1}\right)}{1-\mathcal{R} e\left(\tau_{n+1} \hat{\alpha}_{n-1}\right)}=\frac{\mathcal{I} m\left(\tau_{n} \hat{\alpha}_{n-1}\right)}{1+\mathcal{R} e\left(\tau_{n} \hat{\alpha}_{n-1}\right)}, \quad n \geq 1 .
$$

From the expression for the Verblunsky coefficients in Theorem 2.1,

$$
1+\tau_{n} \hat{\alpha}_{n-1}=\frac{2 \mathfrak{m}_{n}}{1-i c_{n+1}} \quad \text { and } \quad 1-\tau_{n+1} \hat{\alpha}_{n-1}=\frac{2\left(1-\mathfrak{m}_{n}\right)}{1+i c_{n+1}} \quad n \geq 1 .
$$

Hence,

$$
\begin{gathered}
\mathfrak{m}_{n}=\frac{1}{2} \frac{\left|1+\tau_{n} \hat{\alpha}_{n-1}\right|^{2}}{1+\mathcal{R} e\left(\tau_{n} \hat{\alpha}_{n-1}\right)}=\frac{1}{2} \frac{1-\left|\tau_{n+1} \hat{\alpha}_{n-1}\right|^{2}}{1-\mathcal{R} e\left(\tau_{n+1} \hat{\alpha}_{n-1}\right)}, \quad n \geq 1, \\
1-\mathfrak{m}_{n}=\frac{1}{2} \frac{1-\left|\tau_{n} \hat{\alpha}_{n-1}\right|^{2}}{1+\mathcal{R} e\left(\tau_{n} \hat{\alpha}_{n-1}\right)}=\frac{1}{2} \frac{\left|1-\tau_{n+1} \hat{\alpha}_{n-1}\right|^{2}}{1-\mathcal{R} e\left(\tau_{n+1} \hat{\alpha}_{n-1}\right)}, \quad n \geq 1,
\end{gathered}
$$

and $d_{n+1}=\left(1-\mathfrak{m}_{n-1}\right) \mathfrak{m}_{n}, n \geq 1$, with $\mathfrak{m}_{0}=0$.

\section{Example}

Let the real sequences $\left\{c_{n}\right\}_{n=1}^{\infty}$ and $\left\{d_{n+1}\right\}_{n=1}^{\infty}$ be given by

$$
c_{n}=\frac{\eta}{\lambda+n}, \quad d_{n+1}=\frac{1}{4} \frac{n(2 \lambda+n+1)}{(\lambda+n)(\lambda+n+1)}, \quad n \geq 1,
$$

where $\lambda, \eta \in \mathbb{R}$ and $\lambda>-1$.

Observe that $d_{n+1}=\left(1-\mathfrak{m}_{n-1}\right) \mathfrak{m}_{n}, n \geq 1$, where

$$
\mathfrak{m}_{n}=\frac{n}{2(\lambda+n+1)}, \quad n \geq 0 .
$$

Hence, with our assumption $\lambda>-1$, the sequence $\left\{d_{n+1}\right\}_{n=1}^{\infty}$ is a positive chain sequence with $\left\{\mathfrak{m}_{n}\right\}_{n=0}^{\infty}$ as its minimal parameter sequence.

The polynomials $R_{n}$ obtained from the above sequences $\left\{c_{n}\right\}$ and $\left\{d_{n+1}\right\}$, together with the recurrence formula (1.2), are

$$
R_{n}(z)=\frac{(2 \lambda+2)_{n}}{(\lambda+1)_{n}}{ }_{2} F_{1}(-n, b+1 ; b+\bar{b}+2 ; 1-z), \quad n \geq 1
$$


where $b=\lambda+i \eta$. The $n$th degree Hypergeometric polynomial ${ }_{2} F_{1}(-n, b+1 ; b+\bar{b}+2 ; 1-z)$ is defined by

$$
{ }_{2} F_{1}(-n, b+1 ; b+\bar{b}+2 ; 1-z)=\sum_{k=1}^{\infty} \frac{(-n)_{k}(b+1)_{k}}{(b+\bar{b}+2)_{k} k !}(1-z)^{k}=\sum_{k=1}^{n} \frac{(-n)_{k}(b+1)_{k}}{(b+\bar{b}+2)_{k} k !}(1-z)^{k},
$$

where $(a)_{0}=1$ and $(a)_{n}=a(a+1) \cdots(a+n-1), n \geq 1$ are the Pochhammer symbols.

With $d_{1} \neq 0$ arbitrary, by considering the series expansion of ${ }_{2} F_{1}(1,-b ; \bar{b}+2 ; z)$ via its Gauss type continued fraction expansion, we have

$$
\frac{Q_{n}(z)}{R_{n}(z)} \sim \frac{2 d_{1}}{1-i c_{1}}{ }_{2} F_{1}(1,-b ; \bar{b}+2 ; z)=-\nu_{1}-\nu_{2} z-\nu_{3} z^{2}-\ldots,
$$

where

$$
\nu_{n}=d_{1} \frac{b+\bar{b}+2}{b+1} \frac{(-b-1)_{n}}{(\bar{b}+1)_{n}}, \quad n \geq 1 .
$$

From these, for the moment functional $\mathcal{N}$ we obtain

$$
\mathcal{N}[\ell(z)]=\int_{\mathcal{C}} \ell(\zeta) d \psi(b ; \zeta)
$$

for all Laurent polynomials $\ell$, where

$$
d \psi(b ; z)=d_{1} \frac{i|\Gamma(b+1)|^{2}(b+\bar{b}+2)}{2 \pi \Gamma(b+\bar{b}+2)}(-z)^{-\bar{b}-1}(1-z)^{b+\bar{b}+1} d z .
$$

Here, the branch cuts in $(-z)^{-\bar{b}}=\left(e^{-i \pi} z\right)^{-\bar{b}}$ and $(1-z)^{b+\bar{b}}=\left(e^{-i \pi}(z-1)\right)^{b+\bar{b}}$ are along the positive real axis.

For the proofs of the above results, starting from (3.8), we cite for example [2] and [4]. However, for the required analysis in [2] and [4], it was necessary to assume that $\lambda>-1 / 2$. We remark that when $\lambda>-1 / 2$ the maximal parameter sequence $\left\{M_{1, n}\right\}_{n=0}^{\infty}$ of $\left\{d_{n+1}=d_{1, n}\right\}_{n=1}^{\infty}$ is

$$
M_{1, n}=\frac{1}{2} \frac{2 \lambda+n+1}{\lambda+n+1}, \quad n \geq 0 .
$$

Now, the nontrivial probability measure $\hat{\mu}$ that follows from Theorem 2.1 is

$$
d \hat{\mu}(\zeta)=\frac{|\Gamma(b+2)|^{2}}{2 \pi \Gamma(b+\bar{b}+3)}(-z)^{-\bar{b}-2}(1-z)^{b+\bar{b}+2} d z .
$$

Observe that (3.11) can also be written in the equivalent form

$$
d \hat{\mu}\left(e^{i \theta}\right)=\frac{2^{b+\bar{b}+2}|\Gamma(b+2)|^{2}}{2 \pi \Gamma(b+\bar{b}+3)} e^{(\pi-\theta) \mathcal{I} m(b+1)}\left[\sin ^{2}(\theta / 2)\right]^{\mathcal{R} e(b+1)} d \theta .
$$

The moments associated with $\hat{\mu}$ are

$$
\bar{\mu}_{-n}=\mu_{n}=\int_{\mathcal{C}} \zeta^{-n} d \hat{\mu}(\zeta)=\frac{1+c_{1}^{2}}{4 d_{1}}\left[\nu_{n}-\nu_{n+1}\right]=\frac{(-b-1)_{n}}{(\bar{b}+2)_{n}}, \quad n=0,1,2, \ldots
$$

Hence, from results established in [7] the associated monic OPUC are

$$
\hat{S}_{n}(z)=\frac{(b+\bar{b}+3)_{n}}{(b+2)_{n}}{ }_{2} F_{1}(-n, b+2 ; b+\bar{b}+3 ; 1-z), \quad n \geq 1 .
$$

The above expression can also be easily derived from (2.3) and (3.8). 
Recall that we have assumed $\lambda>-1$ and for the integral $I=\int_{\mathcal{C}}(\zeta-1)^{-1} \zeta d \hat{\mu}(\zeta)$ we have from (3.9) and (3.10) that

$$
I=\frac{\bar{b}+1}{b+\bar{b}+2}
$$

Note that when $\lambda>-1 / 2$ the maximal parameter sequence $\left\{M_{1, n}\right\}_{n=0}^{\infty}$ of $\left\{d_{n+1}\right\}_{n=1}^{\infty}$ is different from its minimal parameter sequence $\left\{\mathfrak{m}_{n}\right\}_{n=0}^{\infty}$. Since the integral $J=\int_{\mathcal{C}} \zeta(1-\zeta)^{-1}(\zeta-$ $1)^{-1} d \hat{\mu}$ exists when $\lambda>-1 / 2$, this is exactly what was established in Theorem 2.1 .

However, when $-1 / 2 \geq \lambda>-1$, the integral $J=\int_{\mathcal{C}} \zeta(1-\zeta)^{-1}(\zeta-1)^{-1} d \hat{\mu}$ does not exist and, hence from Theorem 2.1, the minimal parameter of $\left\{d_{n+1}\right\}_{n=1}^{\infty}$ must be equal to its maximal parameter sequence. This we can verify as follows.

From (3.7) we have

$$
\sum_{n=1}^{\infty}\left[\prod_{k=1}^{n} \frac{\mathfrak{m}_{k}}{1-\mathfrak{m}_{k}}\right]=\sum_{n=1}^{\infty} \frac{(1)_{k}}{(2 \lambda+3)_{k}} .
$$

The infinite series on the right hand side can also be written as

$$
{ }_{2} F_{1}(1,1 ; 2 \lambda+3 ; 1)=\sum_{k=0}^{\infty} \frac{(1)_{k}(1)_{k}}{(2 \lambda+3)_{k} k !},
$$

which is (see [1, p. 62]) convergent if $\lambda>-1 / 2$ and divergent otherwise. Thus, from Wall's criteria for maximal parameter sequences we establish that the minimal parameter sequence $\left\{\mathfrak{m}_{n}\right\}$ is also the maximal parameter sequence if $-1 / 2 \geq \lambda>-1$.

\section{References}

[1] G.E. Andrews, R. Askey and R. Roy, "Special Functions", Encyclopedia of Mathematics and its Applications, Cambridge University Press, 2000.

[2] K. Castillo, M.S. Costa, A. Sri Ranga and D.O. Veronese, A Favard type theorem for orthogonal polynomials on the unit circle from a three term recurrence formula, submitted.

[3] T.S. Chihara, "An Introduction to Orthogonal Polynomials", Mathematics and its Applications Series, Gordon and Breach, 1978.

[4] M.S. Costa, H.M. Felix and A. Sri Ranga, Orthogonal polynomials on the unit circle and chain sequences, J. Approx. Theory, 173 (2013), 14-32.

[5] D.K. Dimitrov and A. Sri Ranga, Zeros of a family of hypergeometric para-orthogonal polynomials on the unit circle, Math. Nachr., 286 (2013), 1778-1791.

[6] M.E.H. Ismail, "Classical and Quantum Orthogonal Polynomials in One Variable", Encyclopedia of Mathematics and Its Aplications, vol. 98, Cambridge Univ. Press, 2005.

[7] A. Sri Ranga, Szegő polynomials from hypergeometric functions, Proc. Amer. Math. Soc., 138 (2010), 4259-4270.

[8] G. Szego", "Orthogonal Polynomials", 4th ed., Amer. Math. Soc. Colloq. Publ., vol. 23, Amer. Math. Soc., Providence, RI, 1975.

[9] H.S. Wall, "Analytic Theory of Continued Fractions", D. van Nostrand, 1948. 